\title{
Isolation of a New Chromene from the Brown Alga Sargassum thunbergii
}

\author{
Youngwan Seo." Ki Eui Park, and Taek Jeong Nam ${ }^{\dagger}$
}

\begin{abstract}
Division of Marine Environment and Bioscience, Korea Maritime University, Busan 606-791, Korea. "E-mail: ywseo@hhu.ac.kr" ${ }^{`}$ Factlty of Food Science and Biotechnology, Pukyong National University, Busan 608-737, Korea Received Jume 29, 2007
\end{abstract}

Key Words : Sargassum thunbergii, Sargathunbergol, Chromene, Antioxidant activity, DPPH

Chromenes related with the tocotrienol are frequently found among the brown algae, ${ }^{1-6}$ particularly abundant within the genus Sargassim. ${ }^{1-4,7}$ Studies on biological activities of tocotrienols revealed that they inhibit the growth of several tumor cells and are also effective against muscle cell proliferation, which plays a key role in atherosclerosis. ${ }^{8}$ The initial event in several human disease processes involving cancer and atherosclerosis is oxidative damage to several cellular components such as lipids, proteins, and DNAs. ${ }^{9}$ This has intensified the search for antioxidant from natural sources.

In the course of our continuous search for novel antioxidant compounds from marine plants, we have recently investigated the secondary metabolites from the brown alga Sargassum thunbergit, leading to the isolation of two new tocotrienols, thunbergols $\mathrm{A}$ and $\mathrm{B} .^{10}$ In addition to thunbergols, careful reexamination of chromatographic fractions of the above alga by 'H NMR spectral analysis exhibited the presence of a structurally related compound as a very minor component. Consequently, we pursued this compound in a large-scale collection followed by its large-scale extraction<smiles>CC(C)=CCC/C(=C/CC/C(C)=C/CC/C(C)=C/Cc1cc(O)cc(C)c1O)C(=O)O</smiles><smiles>CC(C)=CCC/C(=C\CC/C(C)=C/CCC1(C)C=Cc2cc(O)cc(C)c2O1)CC/C=C(\C)CCC/C(C)=C/CC1=CC(=O)C=C(C)C1=O</smiles><smiles>CC(=CCCCC1(F)C=Cc2cc(O)cc(C)c2O1)CCC=C(C)CCC=C(CCC(O)C(C)(C)O)C(=O)O</smiles>

Figure 1. Chemical Structure of Compounds 1-5. and a bioactivity-guided separation. In this paper we report the structure and bioactivity of sargathunbergol A (4), a novel chromene.

The brown alga was collected by hand off the shore of Youngdo Island, in Busan. The lyophilized specimens were repeatedly extracted with a mixture of acetone and methylene chloride (1:1), and methanol. The combined crude extracts were separated by solvent-partitioning and $\mathrm{C}_{18}$ vacuum flash chromatography, guided by the results of antioxidant activity and ${ }^{1} \mathrm{H} N \mathrm{NR}$ spectral analysis.

The structures of the major metabolites, sargahydroquinoic acid $(1),{ }^{2}$ sargaquinoic acid $(2),{ }^{1,8}$ and sargachromenol $(3)^{1,8}$ were readily determined by comparison of spectral data with those obtained previously by this group and others. Concentrations of these compounds were an order of magnitude higher than other metabolite. Sargathunbergol (4) A was isolated as a colorless gum, whose molecular weight was deduced as $\mathrm{C}_{27} \mathrm{H}_{38} \mathrm{O}_{6}$ by $\mathrm{MS}$ and ${ }^{13} \mathrm{C} \mathrm{NMR}$ spectrometry. This compound showed close resemblance to the spectroscopic data of 3 . The presence of a chromene ring
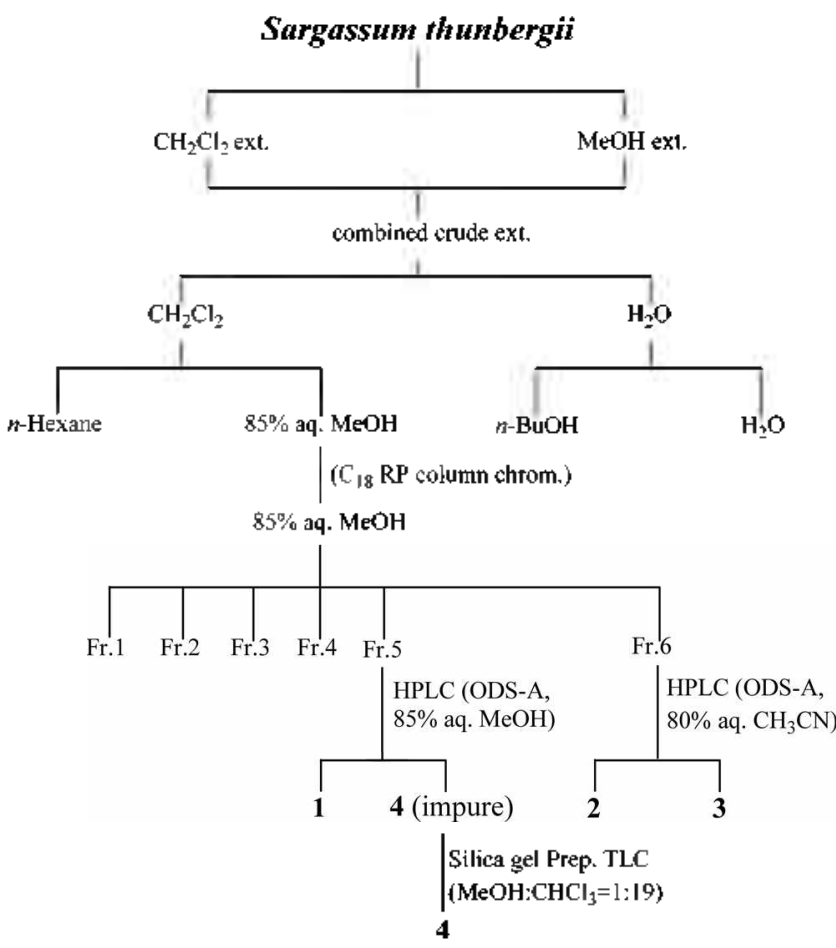

Scheme 1. Isolation of Compounds 1-3 from Sargassum thunbergii. 
was revealed by an occurrence of several carbon signals in the region of $\delta 150-110$ in the ${ }^{13} \mathrm{C}$ NMR spectrum and their corresponding proton signals in the 'H NMR spectrum. Particularly helpful to define it were two doublets at $\delta 6.21$ $(1 \mathrm{H}, \mathrm{d}, J=9.9 \mathrm{~Hz})$ and $5.54(1 \mathrm{H}, \mathrm{d}, J=9.9 \mathrm{~Hz})$ in the ${ }^{\prime} \mathrm{H}$ NMR spectrum, which were typical of the chromene moiety of C-3 unsaturated tocotrienols and assigned to its $\mathrm{H}-3$ and $\mathrm{H}-4$, respectively. Also, the presence of three oxygenated carbons was recognized by signals at $\delta 85.7(\mathrm{CH}), 77.6(\mathrm{C})$ and $71.5(\mathrm{C})$ in ${ }^{13} \mathrm{C}$ NMR spectrum, and a signal at $\delta 4.04$ $(1 \mathrm{H}, \mathrm{dd}, J=11.6 \mathrm{~Hz}, 2.8 \mathrm{~Hz})$ in 'H NMR spectrum.

With the aid of this information, the structure of compound 4 was determined by a combined 2-D NMR analysis. The presence of 2-alkyl-2,8-dimethyl-2H-chromen-6-ol moiety was readily recognized by long-range correlations of downfield protons at $\delta 6.39(1 \mathrm{H}, J=2.8 \mathrm{~Hz}), 6.24(1 \mathrm{H}, J=$ $2,8 \mathrm{~Hz}), 6.16(1 \mathrm{H}, \mathrm{d}, J=9.9 \mathrm{~Hz})$, and $5.49(1 \mathrm{H}, \mathrm{d}, J=9.9$ $\mathrm{Hz}$ ) and upfield protons at $\delta 1.29$ with adjacent carbons in the HMBC experiment. Similarly, long-range correlations of the methyl protons at $\delta 1.50,1.20$, and 1.15 , with neighboring carbons, combined with the ${ }^{1} \mathrm{H}$ COSY correlations of the olefinic and oxymethine protons with upfield protons, defined the structure of the linear prenyl portion bearing a carboxy group at $\mathrm{C}-8^{\prime}$ and two hydroxyl groups at $\mathrm{C}-10^{\circ}$ and $-11^{\prime}$, respectively. Comparison of NMR spectral data for 4 with those for 5 supported this interpretation. ${ }^{11.12}$ In ${ }^{13} \mathrm{C}$ NMR spectrum, resonances of the C-13, $-14,-15,-16$, and -17 carbons of 5 were found at $\delta 23.5,87.1,71.9,26.0$, and 24.9 , respectively, which were very similar to those of the C$10^{\prime},-11^{\prime},-12^{\prime},-13^{\prime}$, and $-14^{\prime}$ carbons of 4 .

Thus, the structure of sargathunbergol was determined as a $10^{\prime}, 11^{\prime}$-dihydroxy derivative of 3 , that is to say, $9-(2,8-$ dimethyl-6-hydroxy-2H-1-benzopyran-2-yl)-6-methyl-2-(4methyl-3,4-dihydroxypentyl)-(2E,6E)-nonadienoic acid.

These compounds are structurally related to the natural antioxidant vitamin $\mathrm{E}$. In our measurement for evaluating the capacity to scavenge the stable free radical of 1,1diphenyl-2-picrylhydrazyl (DPPH), compounds 1-4 exhibited a $\mathrm{EC}_{50}$ value of $20,27,32$, and $38 \mathrm{\mu g} / \mathrm{mL}$, in comparison with reference antioxidants $\mathrm{BHT}\left(\mathrm{EC}_{50}, 42 \mu \mathrm{g} / \mathrm{mL}\right)$ and $\alpha$ tocopherol $\left(\mathrm{EC}_{50}, 23 \mu \mathrm{g} / \mathrm{mL}\right)$.

\section{Experimental Section}

General. NMR spectra were recorded in $\mathrm{CDCl}_{3}$ on a Varian Mercury 300 spectrometer $\left(300 \mathrm{MHz}\right.$ for ${ }^{1} \mathrm{H}$ and 75.5 $\mathrm{MHz}$ for ${ }^{13} \mathrm{C}$ ) using standard pulse sequence programs. All chemical shifts were recorded in $\delta$ values and in ppm with respect to TMS as an internal standard. Mass spectral data were obtained at the Korean Basic Science Institute, Taejeon, Korea. High performance liquid chromatography (HPLC) was performed with a Dionex P580 with Varian 350 RI detector. Column chromatography was done with a RP18 (YMC-GEL ODS-A, $12 \mathrm{~nm}, \mathrm{~S}-75 \mu \mathrm{M}$ ), HP20 (Diaion HP-20, SUPELCO, USA), and Silica gel G (Merck). All solvents used were spectral grade or were distilled in glass prior to use.
Table 1. NMR Spectral Assignments for Sargathunbergol (4)

\begin{tabular}{|c|c|c|c|c|}
\hline \multirow{2}{*}{ no } & \multicolumn{2}{|l|}{3} & \multicolumn{2}{|l|}{4} \\
\hline & $\mathrm{H}$ & $\mathrm{C}$ & $\mathrm{H}$ & $\mathrm{C}$ \\
\hline 2 & & $77.7 \mathrm{~s}$ & & $77.6 \mathrm{~s}$ \\
\hline 3 & $5.54(1 \mathrm{H}, \mathrm{d}, 9.6)$ & $130.5 \mathrm{~d}$ & $5.54(1 \mathrm{H}, \mathrm{d}, 9.9)$ & $130.6 \mathrm{~d}$ \\
\hline 4 & $6.21(1 \mathrm{H}, \mathrm{d}, 9.6)$ & $122.8 \mathrm{~d}$ & $6.21(1 \mathrm{H}, \mathrm{d}, 9.9)$ & $122.8 \mathrm{~d}$ \\
\hline $4 a$ & & $121.2 \mathrm{~s}$ & & $121.2 \mathrm{~s}$ \\
\hline 5 & $6.30(1 \mathrm{H}, \mathrm{d}, 2.2)$ & $110.3 \mathrm{~d}$ & $6.29(1 \mathrm{H}, \mathrm{d}, 2.8)$ & $110.2 \mathrm{~d}$ \\
\hline 6 & & $148.5 \mathrm{~s}$ & & $148.5 \mathrm{~s}$ \\
\hline 7 & $6.45(1 \mathrm{H}, \mathrm{d}, 2.2)$ & $117.0 \mathrm{~d}$ & $6.44(1 \mathrm{H}, \mathrm{d}, 2.8)$ & $117.0 \mathrm{~d}$ \\
\hline 8 & & $126.2 \mathrm{~s}$ & & $126.2 \mathrm{~s}$ \\
\hline $8 a$ & & $144.6 \mathrm{~s}$ & & $144.7 \mathrm{~s}$ \\
\hline $1^{\dagger}$ & $1.65(2 \mathrm{H}, \mathrm{m})$ & $40.8 \mathrm{t}$ & $1.62(2 \mathrm{H}, \mathrm{m})$ & $40.8 \mathrm{t}$ \\
\hline $2^{+}$ & $2.11(2 \mathrm{H}, \mathrm{m})$ & $22.7 \mathrm{t}$ & $2.07(2 \mathrm{H}, \mathrm{m})$ & $22.7 \mathrm{t}$ \\
\hline $3^{+}$ & $5.12(1 \mathrm{H}, \mathrm{t}, 6.9)$ & $124.8 \mathrm{~d}$ & $5.10(1 \mathrm{H}, \mathrm{t}, 6.3)$ & $124.9 \mathrm{~d}$ \\
\hline $4^{r}$ & & $134.2 \mathrm{~s}$ & & $134.1 \mathrm{~s}$ \\
\hline $5^{\prime}$ & $2.05(2 \mathrm{H}, \mathrm{t}, 7.5)$ & $39.1 \mathrm{t}$ & $2.05(2 \mathrm{H}, \mathrm{m})$ & $38.8 \mathrm{t}$ \\
\hline $6^{r}$ & $\begin{array}{l}2.58(2 \mathrm{H}, \mathrm{dt} \\
7.0,7.5)\end{array}$ & $28.2 \mathrm{t}$ & $2.67(2 \mathrm{H}$, br q, 7.2$)$ & $28.0 \mathrm{t}$ \\
\hline 7 & $5.96(1 \mathrm{H}, \mathrm{t}, 7.0)$ & $145.4 \mathrm{~d}$ & $6.00(1 \mathrm{H}, \mathrm{t}, 6.9)$ & $147.9 \mathrm{~d}$ \\
\hline $8^{+}$ & & $130.4 \mathrm{~s}$ & & $124.1 \mathrm{~s}$ \\
\hline $9^{+}$ & $2.25(2 \mathrm{H}, \mathrm{t}, 6.9)$ & $34.6 \mathrm{t}$ & $2.46(2 \mathrm{H}, \mathrm{m})$ & $28.7 \mathrm{t}$ \\
\hline $10^{\dagger}$ & $2.11(2 \mathrm{H}, \mathrm{m})$ & $27.9 \mathrm{t}$ & $\begin{array}{l}1.94(1 \mathrm{H}, \mathrm{m}) \\
1.65(1 \mathrm{H}, \mathrm{m})\end{array}$ & $23.6 \mathrm{t}$ \\
\hline $11^{\prime}$ & $5.07(1 \mathrm{H}, \mathrm{t}, 6.9)$ & $123.3 \mathrm{~d}$ & $4.04(1 \mathrm{H}, \mathrm{dd}, 11.6,2.8)$ & $85.7 \mathrm{~d}$ \\
\hline $12^{+}$ & & $132.1 \mathrm{~s}$ & & $71.5 \mathrm{~s}$ \\
\hline $13^{r}$ & $1.66(3 \mathrm{H}, \mathrm{s})$ & $25.7 q$ & $1.25(3 \mathrm{H}, \mathrm{s})$ & $25.8 \mathrm{q}$ \\
\hline $14^{r}$ & $1.57(3 \mathrm{H}, \mathrm{s})$ & $17.8 \mathrm{q}$ & $1.20(3 \mathrm{H}, \mathrm{s})$ & $24.3 \mathrm{q}$ \\
\hline $15^{r}$ & & $172.9 \mathrm{~s}$ & & $165.4 \mathrm{~s}$ \\
\hline 16 & $1.56(3 \mathrm{H}, \mathrm{s})$ & $15.6 \mathrm{q}$ & $1.55(3 \mathrm{H}, \mathrm{s})$ & $15.8 \mathrm{q}$ \\
\hline $17^{r}$ & $1.35(3 \mathrm{H}, \mathrm{s})$ & $26.0 \mathrm{q}$ & $1.34(3 \mathrm{H}, \mathrm{s})$ & $26.0 \mathrm{q}$ \\
\hline $18^{\prime}$ & $2.11(3 \mathrm{H}, \mathrm{s})$ & $15.8 \mathrm{q}$ & $2.11(3 \mathrm{H}, \mathrm{d}, 1.4)$ & $15.6 \mathrm{q}$ \\
\hline
\end{tabular}

All assignments were based on DEPT, COSY, TOCSY, HMQC, HMBC, and NOESY experiments.

Collection, extraction, and isolation. Sargassum thunbergii (sample number 06PK-3) was collected by hand in March 2006 , along the shore of Youngdo Island, South Sea, Korea. The collected samples were briefly dried under shade and kept at $-25^{\circ} \mathrm{C}$ until chemically investigated. Samples $(0.5$ $\mathrm{kg}$ ) of Sargassum thunbergi $i$ were chopped into small pieces and extracted for 2 days with $\mathrm{MeOH}(3 \mathrm{~L} \times 2)$ and $\mathrm{CH}_{2} \mathrm{Cl}_{2}(3$ $\mathrm{L} \times 2)$, respectively. The combined crude extracts $(29.8 \mathrm{~g})$ were evaporated under reduced pressure and suspended in $\mathrm{H}_{2} \mathrm{O}$ and then partitioned with $\mathrm{CH}_{2} \mathrm{Cl}_{2}$. The organic layer was further partitioned between $85 \%$ aq. $\mathrm{MeOH}$ and $n$ hexane. A portion of the lower layer $(8.2 \mathrm{~g})$ was subjected to $\mathrm{C}_{18}$ reversed-phase vacuum flash chromatography using stepwise gradient mixtures of $\mathrm{MeOH}$ and water $(50 \%, 60 \%$, $70 \%, 80 \%, 90 \%$ aq. $\mathrm{MeOH}$, and $100 \% \mathrm{MeOH}$ ) as eluents. Purification of fraction 5 by semi-preparative $\mathrm{C}_{18}$ HPLC (YMC ODS-A column, $85 \%$ aq. $\mathrm{MeOH}, 1 \mathrm{~cm} \times 25 \mathrm{~cm}, 2$ $\mathrm{mL} / \mathrm{min})$ gave compounds $1(33.7 \mathrm{mg})$ and 4. Further purification of $\mathbf{4}$ was made by Prep. TLC on a silica gel plate with $\mathrm{MeOH} / \mathrm{CHCl}_{3}(1: 19)$ as a solvent system to yield $4.3 \mathrm{mg}$ of 4 . Fraction 6 was subjected to reversedphase HPLC (YMC ODS-A, $80 \%$ aq. MeCN, $1 \mathrm{~cm} \times 25 \mathrm{~cm}$, $2 \mathrm{~mL} / \mathrm{min}$ ) to give $36.2 \mathrm{mg}$ and $40.2 \mathrm{mg}$ of 2 and 3 , 
respectively.

Sargahydroquinoic acid (1): colorless gum; FABMS $(\mathrm{M}+\mathrm{Na})^{+} m / z 449$.

Sargaquinoic acid (2): colorless gum; FABMS $(\mathrm{M}+\mathrm{Na})^{+}$ $m / z 447$.

Sargachromenol (3): colorless gum; FABMS (M+Na) ${ }^{+} m / z$ 447.

Sargathunbergol (4): colorless gum; $[\alpha]_{\mathrm{D}}^{25}+2.8^{\circ}(\mathrm{c} 0.05$, $\mathrm{MeOH}) ;{ }^{1} \mathrm{H}$ - and ${ }^{13} \mathrm{C}-\mathrm{NMR}$ spectral data, see Table 1; $\mathrm{HMBC}$ correlations $\mathrm{H}-4 / \mathrm{C}-8 \mathrm{a}, \mathrm{C}-5 ; \mathrm{H}-3 / \mathrm{C}-2, \mathrm{C}-4 \mathrm{a} ; \mathrm{H}-2 / \mathrm{C}-$ $3^{\prime}, \mathrm{C}-4^{\prime} ; \mathrm{H}-3^{\prime} / \mathrm{C}-5^{\prime}, \mathrm{C}-16^{\prime} ; \mathrm{H}-5^{\prime} / \mathrm{C}-3^{\prime}, \mathrm{C}-4^{\prime}, \mathrm{C}-5^{\prime}, \mathrm{C}-16^{\prime} ; \mathrm{H}-6^{\prime} /$ $\mathrm{C}-4^{\prime}, \mathrm{C}-5^{\prime}, \mathrm{C}-6^{\prime}, \mathrm{C}-7^{\prime}, \mathrm{C}-8^{\prime} ; \mathrm{H}-7^{\prime} / \mathrm{C}-5^{\prime}, \mathrm{C}-9^{\prime}, \mathrm{C}-15^{\prime} ; \mathrm{H}-13^{\prime} / \mathrm{C}-$ $11^{\prime}, \mathrm{C}-12^{\prime}, \mathrm{C}-14^{\prime} ; \mathrm{H}-14^{\prime} / \mathrm{C}-11^{\prime}, \mathrm{C}-12^{\prime}, \mathrm{C}-13^{\prime} ; \mathrm{H}-16 / \mathrm{C}-3{ }^{\prime}, \mathrm{C}-4$, $\mathrm{C}-5 ;$; $-17 / \mathrm{C}-2, \mathrm{C}-3, \mathrm{C}-1$; H-5/C-7, C-8a; H-7/C-5, C-8a; $\mathrm{H}-18 / \mathrm{C}-7, \mathrm{C}-8, \mathrm{C}-8 \mathrm{a}$; HRFABMS $\left(\mathrm{M}+\mathrm{Na}-\mathrm{H}_{2} \mathrm{O}\right)^{+} \mathrm{m} / \mathrm{z}$ obsd 463.2560 (Calcd for $\mathrm{C}_{27} \mathrm{H}_{36} \mathrm{O}_{5} \mathrm{Na}, 463.2460$ ); ESIMS (M$\mathrm{H})^{+} m / z 457$.

Acknowledgements. Mass spectral data were kindly provided by Korea Basic Science Institute. This research was supported by a grant (M2007-03) from the Marine Bioprocess Research Center of the Marine Bio 21 Center funded by the Ministry of Maritime Affairs \& Fisheries, Republic of Korea.

\section{References}

I. Kusumi, T.; Shibata, Y; Ishitsuka, M.; Kinoshita, T.; Kakisawa, H. Chem, Lett. 1979, 277.

2. Segawa, M.; Shirahama, H. Chem, Lett, 1987, 1365.

3. Numata, A.; Kanabara, S.; Takahashi, C.; Fujiki, R.; Yoneda, M.; Fujita, E.; Nabeshima. Y. Chem. Pharn. Bull. 1991, 30, 2129.

4. Numata, A.; Kanabara, S.; Takahashi, C.; Fujiki, R.; Yoneda, M.; Usami, Y.; Fujita, E. Phytochemistry 1992, 31, 1209.

5. Mesguiche, V.; Valls, R.; Piovetti, L.; Banaigs, B. Phytochemistry: $1997,45,1489$.

6. Davyt, D.; Enz, W.; Manta, E.; Navarro, G.; Notre, M. Natural Product Letters 1997, 9, 305.

7. Jang. K. H.; Lee, B. H.; Choi, B. W.; Lee, H.-S.; Shin, J. J. Nat. Prods. 2005, 68, 716.

8. Silva, D. H. S.; Pereira, F. C.; Zanoni, M. V. B.; Yoshida, M. Phytochemistry 2001, 57,437.

9. Gordon, M. H. Nat. Prod. Rep. 1996, 13, 265.

10. Seo, Y.; Park, K. E.; Kim, Y. A.; Lee, H.-J.; Yoo, J.-S. Chent. Pharm. Bull. 2006, 54, 1730.

I1. Saad, J. R.; Pestchanker, M. J.; Giordano, O. S. Phytochemistry $1987,26,3033$.

12. Abdel-Saltar, E. Monatsh, Chem, 2001, 132, 1095. 